\title{
Universal processes in heliophysics
}

\author{
Joseph M. Davila ${ }^{1}$, Nat Gopalswamy ${ }^{2}$ and Barbara J. Thompson ${ }^{3}$ \\ ${ }^{1}$ Goddard Space Flight Center, Code 670, Greenbelt, MD 20771, USA \\ email: Joseph.M.Davila@nasa.gov \\ ${ }^{2}$ Goddard Space Flight Center, Code 695, Greenbelt, MD 20771, USA \\ email: Nat.Gopalswamy@nasa.gov \\ ${ }^{3}$ Goddard Space Flight Center, Code 671, Greenbelt, MD 20771, USA \\ email: Barbara.J.Thompson@nasa.gov
}

\begin{abstract}
The structure of the Universe is determined primarily by the interplay of gravity which is dominant in condensed objects, and the magnetic force which is dominant in the rarefied medium between condensed objects. Each of these forces orders the matter into a set of characteristic structures each with the ability to store and release energy in response to changes in the external environment. For the most part, the storage and release of energy proceeds through a number of Universal Processes. The coordinated study of these processes in different settings provides a deeper understanding of the underlying physics governing Universal Processes in astrophysics.
\end{abstract}

Keywords. Sun: general, interplanetary medium, solar system: general

\section{Introduction}

The solar system provides us with our best opportunity to study astrophysics in detail. In-situ observations provide measurements of particle distribution functions for both thermal and energetic particles. Details of these distributions can provide important information on the origin of the plasma. Planetary probes have provided detailed observations of planets which fifty years ago could only be studied by telescopic observation. Present day remote sensing observations both from the ground and from spacecraft provide images with resolution at a scale that cannot be approached for objects outside the Solar System, e.g. the Sun is the only star where the surface and sub-surface features can be resolved and studied. This wealth of detail allows the observation of processes in sufficient detail to identify the underlying physics involved. The processes provide the road map for understanding more distant objects in the Universe.

\section{Physical Origin of the Universal Process Concept}

The large scale structure of the universe is controlled mainly by two forces, gravitation and magnetism. Gravitation is the dominant force controlling the evolution of dense matter in the universe, including planets, stars, planetary systems, galaxies, and clusters of galaxies. Magnetism, a second long-range force, is dominant in the rarefied, ionized matter which generally occupies the space between gravitationally condensed regions. The most easily observed example is the plasma environment of the solar system where the magnetic field is responsible for the storage and subsequent release of large quantities of energy in solar flares, coronal mass ejections (CMEs), magnetic storms, and other transient phenomena. In addition the magnetic field of solid bodies like the Earth, Jupiter, Saturn, and even the Sun, dominate and define the structure of the space environment surrounding them, and exhibit their own energetic evolution. 
A common property of both gravitationally and magnetically structured objects is their ability to store, release, and transfer energy in response to internal and external drivers. For example thunderstorms generate atmospheric gravity waves that transfer energy into the stratosphere, the magnetosphere of Earth continually adjusts in response to the variable solar wind, and the magnetic field of the Sun is constantly buffeted by convective motions in the photosphere.

To illustrate this point, consider an isolated magnetic loop like that shown in the left panel in Figure 1. The magnetic field of the Sun is largely contained in individual loops which maintain their identity while interacting with the surrounding corona. Typically these loops are potential, i.e. current free, which is the lowest energy state for the structure. Convective motions at the surface of the Sun twist and deform the loop causing it to store energy. When the stored energy reaches a large enough level, rapid energy release can be triggered returning the loop to its potential state, resulting in the production of plasma flows, particle acceleration, waves, or heating. These are illustrated schematically in Figure 2.
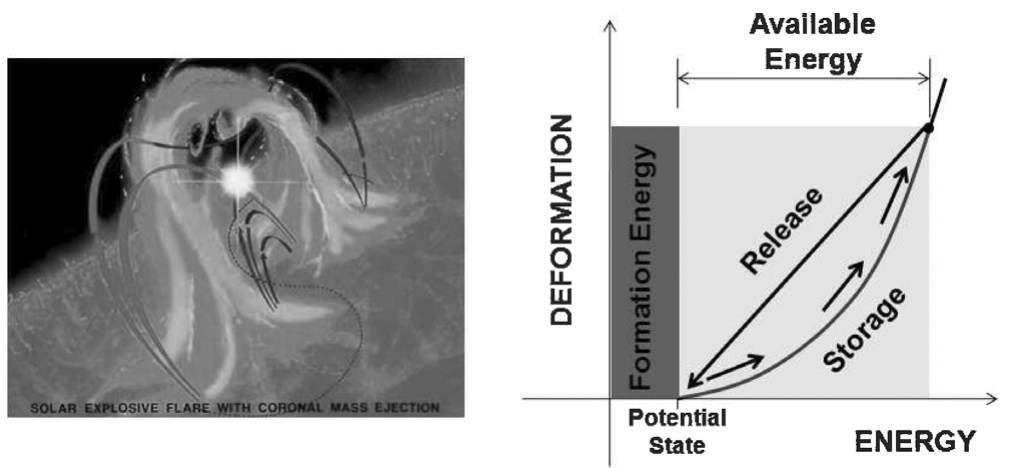

Figure 1. The simplest magnetic structure is a single loop (left panel). On the Sun convective motions shuffle the footpoints of the loop magnetic field generating currents in the corona that store energy (right panel). Eventually this energy is released in other forms.

The evolution of magnetic structures, and planetary atmospheres in the solar system proceeds through a set of Universal Processes (Crooker 2004), e.g. reconnection, particle acceleration, wave generation and propagation, etc. Energy is stored in magnetic structures on the Sun, in Earth's magnetosphere, the magnetospheres of other planets, and in the heliosphere itself. Each of these structures evolves in a similar way. By studying these Universal Processes together, in diverse environments, and in a comparative way, new scientific insights will be gained, and a deeper understanding of the underlying physics can be obtained. These processes are evident in astrophysical settings as well. The study of Universal Processes in heliophysics provides a sound physical basis for the interpretation of astrophysical phenomena.

\section{Universal Processes in the IHY}

The study of Universal Processes is a primary focus of the International Heliophysical Year (IHY). IHY has three primary objectives, (1) Advancing our understanding of the fundamental heliophysical processes that govern the Sun, Earth and heliosphere; (2) Continuing the tradition of international research and advancing the legacy on the 50th anniversary of the International Geophysical Year; (3) Demonstrating the beauty, relevance and significance of space and Earth science to the world. To accomplish these 

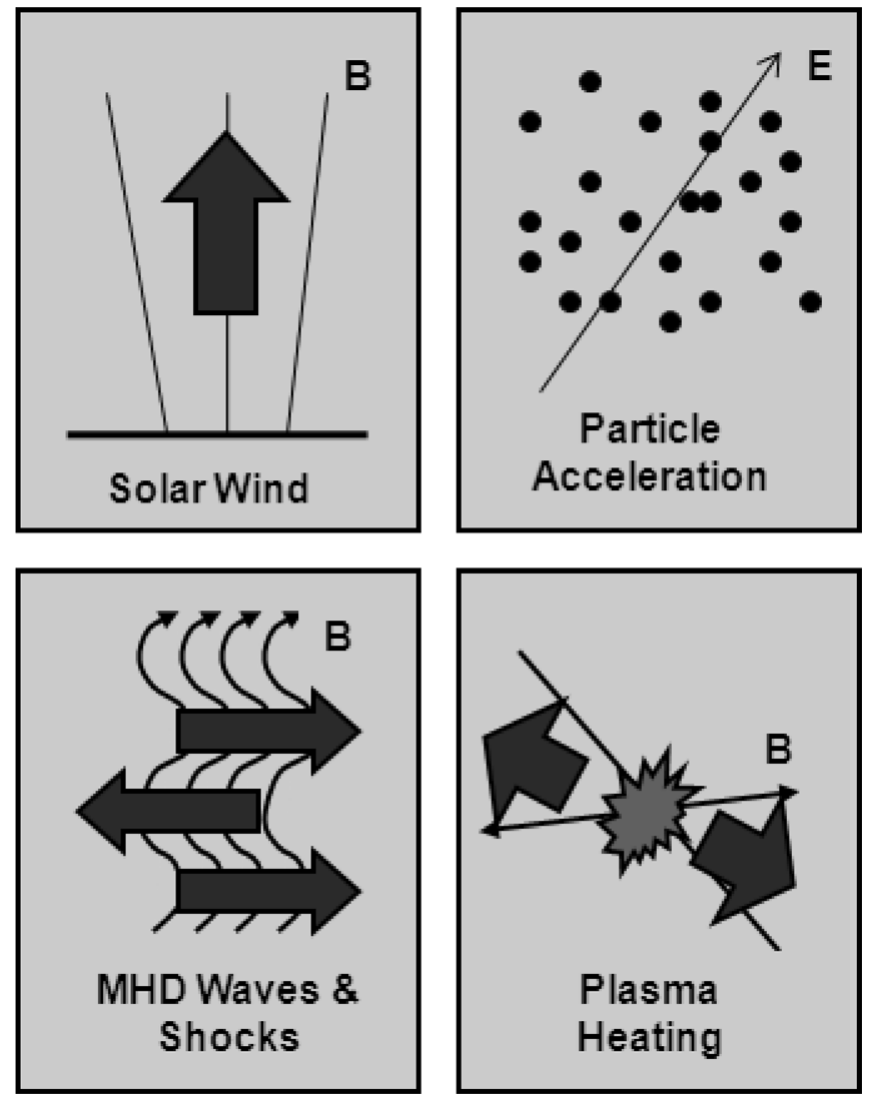

Figure 2. The energy stored in magnetic structures can be released as kinetic energy (upper left), energetic particles (upper right), waves (lower left), or heating (lower right).

objectives, we have identified six goals of IHY, each corresponding to a unique opportunity afforded by IHY:

1. Develop the basic science of heliophysics through cross-disciplinary studies of Universal Processes.

2. Determine the response of terrestrial and planetary magnetospheres and atmospheres to external drivers.

3. Promote research on the Sun-heliosphere system outward to the local interstellar medium - the new frontier.

4. Foster international scientific cooperation in the study of heliophysical phenomena now and in the future.

5. Preserve the history and legacy of the IGY on its 50th Anniversary.

6. Communicate unique IHY results to the scientific community and the general public. IHY is an integrated program of many diverse activities working on an international level to achieve all of the above goals.

\section{Examples of Universal Processes}

The concept of Universal Processes is perhaps best understood by considering a few examples: (1) Shocks are observed in situ in the interplanetary medium, shocks are 
believed to play a role in the acceleration of particles in the solar corona, and standing bow shocks and termination shocks separate the major regions in the heliosphere. Shock formation, and particle acceleration are universal processes. (2) Aurorae are observed on Earth, Saturn, and Jupiter, and Jovian auroral "footprints" have been observed on Io, Ganymede and Europa. The formation of aurorae is observed to be the universal response of a magnetized body in the solar wind. Here we consider two examples of Universal Processes in further detail, magnetic reconnection and atmospheric gravity waves.

\subsection{Magnetic Reconnection}

Magnetic reconnection is one of the best examples of a Universal Process in heliophysics. On the Sun solar flares result from reconnection in the corona (e.g., Chen et al. 2007). Energy stored in the coronal magnetic field is released as energetic particles, flows, and plasma heating. Figure 3 (after Sui and Holman 2003) shows shows an observation from the RHESSI mission. The flaring region is observed in three different spectral passbands, 6-8, 10-12, and 16-20 keV. Two distinct emission regions are seen. In each region the hottest emission is seen to occur nearest the the center of a line connecting the two emission regions. This emission pattern is consistent with reconnection jets emanating from a central point as indicated in the figure.

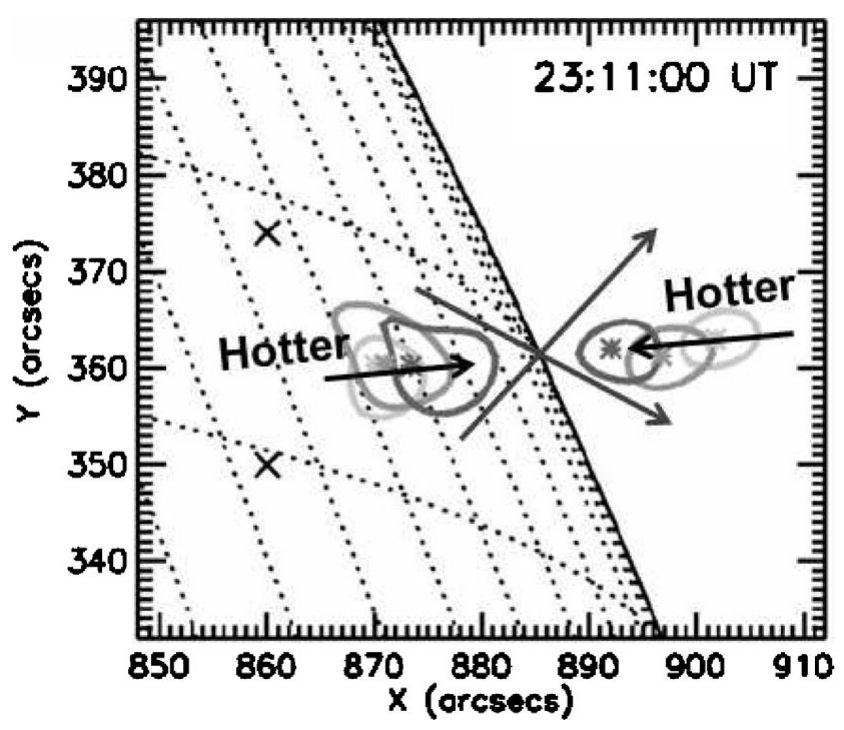

Figure 3. The RHESSI mission observed a flaring region in three passbands, 6-8, 10-12, and 16-20 keV. The spatial positions of the observed regions form a distinct pattern with the hottest to coolest regions symmetrically placed around a central point as would be expected for reconnection (from Sui and Holman 2003). The x-point, arrows, and notations were added for clarity.

Reconnection is also observed in the magnetosphere of Earth (Angelopoulos et al. 2007). The THEMIS mission consists of five spacecraft (Figure 4) providing in-situ measurements in the magnetotail supported by extensive simultaneous ground-based observation of auroral activity. With this powerful combination, the THEMIS team was able to demonstrate for the first time that reconnection observed in the tail resulted in substorm activity in the polar region. 


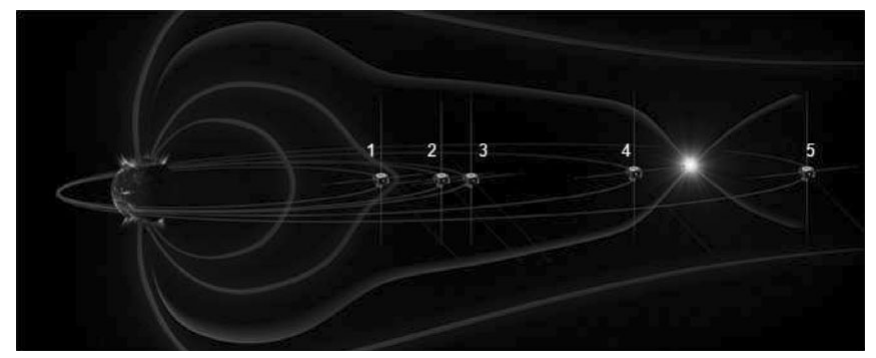

Figure 4. The THEMIS mission consists of five spacecraft orbiting Earth at various distances. This constellation was able to observe magnetic reconnection in the magnetotail while simultaneously viewing substorm activity from the ground.

\subsection{Planetary Gravity Waves}

Universal processes are not confined to the ionized plasma component in the Solar System. The Earth's ionosphere is the boundary between the neutral atmosphere and space (Taylor et al. 1987). Energy between these two components is exchanged through this boundary. One process by which this energy exchange takes place is through atmospheric gravity waves. Gravity waves are excited in the neutral atmosphere by thunderstorms, mountain ranges, and other processes. These waves propagate upward from their point of origin steepening as they propagate. Eventually these waves become non-linear and "break" like ocean waves on a beach depositing their energy in the ionospheremesosphere, providing a potentially important coupling between the troposphere where climatic effects are evident and the magnetosphere. Figure 5 shows several images of gravity waves in both Earth's and Mars' atmospheres. The potential importance of these processes for energy transfer has only recently been recognized, and much additional work remains to establish the significance of the Universal Process of gravity wave propagation on the structure of Earth, Mars, and other planetary atmospheres.
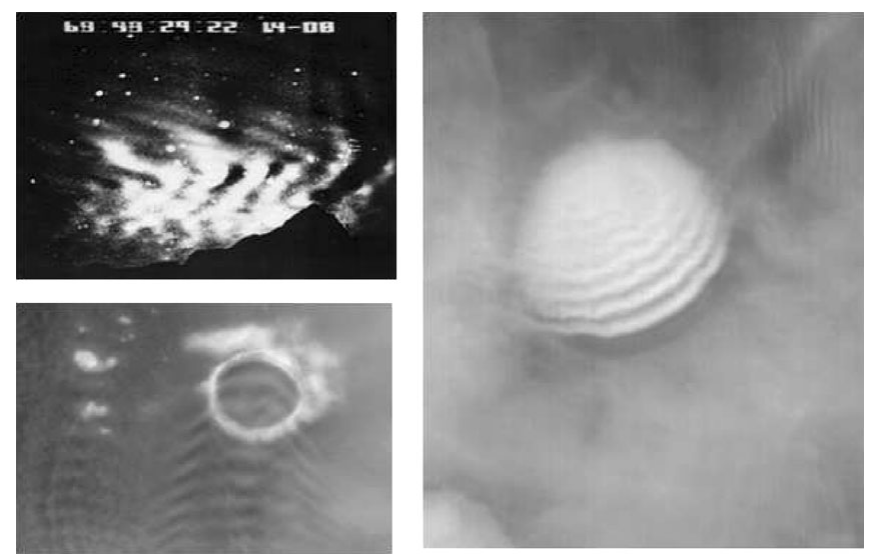

Figure 5. Gravity waves in Earth's atmosphere [upper left panel] transfer energy from the troposphere to the ionosphere-mesosphere (from Taylor et al. 1987). Similar waves seen on Mars by the Mars Global Surveyor Mission (lower left and right panels) may couple the lower and upper Martian atmosphere. 


\section{Summary and Conclusions}

The matter in the Solar System is organized primarily by gravitional and magnetic forces. These forces cause the formation of structures that evolve primarily through Universal Processes. Heliophysics, which is the study of Universal Processes in diverse environments, can lead to a deeper physical understanding of magnetic processes like reconnection, particle acceleration, and the generation of waves and other processes in the neutral atmosphere. Heliophysical processes are the foundation for understanding the physical conditions in astrophysical objects, and as such are essential for our understanding of the Universe.

\section{References}

Angelopoulos, V., McFadden, J. P., Larson, D., Carlson, C. W., Mende, S. B., Frey, H., Phan, T., Sibeck, D. G., Glassmeier, K.-H., Auster, U., Donovan, E., Mann, I. R., Rae, I. J., Russell, C. T., Runov, A., Zhou, X.-Z., \& Kepko, L. 2007,Sci., 321, 931

Chen, P. F., Liu, W. J., \& Fang, C. 2007,Adv. Sp. Res., 39, 1421

Crooker, N. U. 2004,EOS, 85(37), 351

Sui, L. \& Holman G. D. 2003,Ap. J., 596, L251

Taylor, M.J., Hapgood, M.A., \& Rothwell, P. 1987, Planet. Space Sci., 35, 413

\section{Discussion}

SPANGLER: You stated that the resistivity in heliospheric plasmas is small. Isn't a more accurate statement that collisional resistivity is small? Collisionless processes could enhance the resistivity and associated dissipation.

DAvila: Yes you are correct. However to get enhanced resistivity the structure must already be evolving through a collisionless process.

BEER: I'm glad that you mentioned the example of atmospheric gravity waves. Universal processes are not reduced to magnetically organized matter but also in gravitationally organized matter.

DAVILA: Absolutely.

GIRISH: What are the early results in the progress of ground-based experiments going on all over the world as a part of IHY started in 2007?

DAVILA: The IHY has approximately 15 instrument projects. These involve generally an instrument donor and an instrument host. Instruments are now operating in South America, Africa and Asia. The project has been well received and is very successful.

Cliver: The universality of heliospheric processes imposes challenges on our understanding of reconnection, where in the magnetosphere one observes electron skin depth scale of "action", which extrapolates to a few $\mathrm{cm}$ in the solar corona. This may require particular conditions to explain the large flaring fluxes of ions and electrons.

DAVILA: There are certainly important differences between reconnection in the magnetosphere and in solar flares. But I believe that the wonderfully detailed in situ observations in the magnetosphere and high resolution images in flares can help us understand reconnection more completely. 